\title{
Voruntersuchungen zur Einsetzbarkeit des Walzprofilierens zur Herstellung bistabiler dünner Bleche
}

\author{
P. Pavliuchenko' • M. Teller ${ }^{1}$. G. Hirt ${ }^{1}$ \\ Eingegangen: 17. Januar 2021 / Angenommen: 20. April 2021 / Online publiziert: 12. Mai 2021 \\ (c) Der/die Autor(en) 2021
}

\section{Zusammenfassung}

Bistabile Bleche verfügen aufgrund der über Umformprozesse eingebrachten Eigenspannungen über zwei stabile Strukturen: Im Transportzustand können sie platzsparend transportiert werden, bevor sie am Einsatzort in eine gestreckte Form entfaltet werden. Die gestreckte Form weißt eine größere Stabilität auf, wenn sie vollständig geschlossen bzw. rohrförmig ist. Da für die Erreichung bistabiler Eigenschaften Bleche mit einer hohen Streckgrenze und geringer Dicke genutzt werden müssen, werden häufig inkrementelle Umformverfahren bei der Herstellung eingesetzt. Obwohl ein inkrementelles Biegen über zwei orthogonale Achsen bereits die Herstellung vollständig geschlossener bistabiler Bleche von hoher Qualität ermöglicht, ist es als nachteilig zu bewerten, dass die maximale Größe einer solchen Struktur durch die Gesenkbreite begrenzt wird. Ziel dieses Artikels ist daher die Untersuchung des Walzprofilierens als alternatives Umformverfahren zur Herstellung bistabiler Blechstrukturen mit nahezu beliebiger Länge. Es werden Ergebnisse mit einem vereinfachten Ersatzmodell präsentiert, sodass der Einfluss der Walzdurchläufe auf die erreichbaren Krümmungsradien vorab simulativ abgeschätzt werden kann. Anschließend werden Walzprofilierversuche an vorab unverformten Blechen und an Blechen mit vorheriger inkrementeller Biegung präsentiert. Die Ergebnisse zeigen, dass durch eine Kombination von inkrementellem Biegen (1. Umformschritt) und anschließendem Walzprofilieren (2. Umformschritt) bistabile Blechstrukturen erzeugt werden können. Die erreichbaren Krümmungsradien hängen von den durchgeführten Walzdurchläufen bzw. dem Abstand zwischen den einzelnen Walzbahnen ab. Es wird somit eine neue Prozessroute zur Herstellung von bistabilen Blechstrukturen mit nahezu beliebiger Länge präsentiert.

P. Pavliuchenko

pavlo.pavliuchenko@ibf.rwth-aachen.de

1 Institut für Bildsame Formgebung (IBF), RWTH Aachen

University, Intzestraße 10, 52056 Aachen, Deutschland 


\title{
Preliminary investigations on the applicability of roll forming for the production of bistable thin sheets
}

\begin{abstract}
Bistable sheets have two stable structures due to the residual stresses introduced via forming processes: In the transport state, they can be transported in a space-saving manner before being unfolded into an elongated shape at the place of use. The elongated form has greater stability when it is completely closed, i.e. tubular. Since sheets with a high yield strength and low thickness must be used to achieve bistable properties, incremental forming processes are often used in manufacturing. Although incremental bending over two orthogonal axes already allows the production of fully closed bistable sheets of high quality, it is considered disadvantageous that the maximum size of such a structure is limited by the die width. The aim of this article is therefore to investigate roll forming as an alternative forming process for the production of bistable sheet structures of almost any length. Results are presented using a simplified model so that the influence of the rolling passes on the achievable radii of curvature can be simulated in advance. Roll forming tests on previously undeformed sheets and on sheets with previous incremental bending are then presented. The results show that bistable sheet structures can be produced by a combination of incremental bending (1st forming step) and subsequent roll forming (2nd forming step). The achievable radii of curvature depend on the rolling passes performed or the distance between the individual rolling passes, respectively. A new process route for the production of bistable sheet structures of almost any length is thus presented.
\end{abstract}

\section{Einleitung}

Bistabile Metallbleche mit einer vollständig geschlossenen Geometrie im entfalteten Zustand und einer aufgerollten Transportgeometrie sind von großem Interesse für den Leichtbau. Solche Strukturen können ohne Gehäuse transportiert und schließlich am Einsatzort entfaltet werden. Die verwendeten Bleche sollten eine relativ geringe Dicke um 0,1 bis $0,2 \mathrm{~mm}$ und eine hohe Streckgrenze von mehr als $600 \mathrm{MPa}$ aufweisen, um bistabile Eigenschaften durch die Einbringung von Eigenspannungen zu ermöglichen [1]. Aus der Literatur ist bereits bekannt, dass sich über das inkrementelle Biegen der Blechhalbzeuge in zwei entgegengesetzte orthogonale Richtungen solche bistabilen Strukturen herstellen lassen [2]. Die in [3] durchgeführten Messungen der Eigenspannungsverteilung über die Dicke der bistabilen Bleche zeigen eine gute qualitative Übereinstimmung zwischen berechneten und gemessenen Werten. Aufgrund der hohen Streckgrenze und der entsprechend hohen Rückfederung wurde bisher ein inkrementeller Biegeprozess mit kleinen Biegeradien $(<10 \mathrm{~mm})$ eingesetzt. Allerdings beschränkt die Breite des Biegewerkzeugs die maximale Länge der finalen bistabilen Struktur.

In diesem Beitrag wird daher die Anwendbarkeit eines Walzprofilierprozesses zur Erzeugung bistabiler Strukturen untersucht. Das Walzprofilieren ist ein Herstellungsprozess, bei dem das Blech kontinuierlich durch eine Reihe konturierter Walzen geführt wird, die hintereinander angeordnet sind, um schrittweise die endgültige Produktform mit einem gewünschten gleichmäßigen Querschnitt zu erreichen [4]. Durch diesen Fertigungsprozess lassen sich also nahezu beliebige Bauteilgrößen erzeugen. Allerdings stellt die hohe Streckgrenze des Materials kombiniert mit der gerin- gen Blechdicke eine wesentliche Herausforderung in dieser Prozessvariante dar, da eine ausreichende Plastifizierung entsprechend kleine Biegeradien benötigt. Die Notwendigkeit kleiner Biegeradien konnte auch bereits für das inkrementelle Biegen nachgewiesen werden [2], wobei in diesem Prozess direkt über die gesamte Blechbreite umgeformt wird und nicht nur eine lokale Umformzone wie beim Walzprofilieren auftritt. Es gilt also zu prüfen, ob bistabile Strukturen durch Walzprofilieren erzeugt und welche finalen Geometrien im entfalteten Zustand erreicht werden können.

In der Arbeit von Groche und Breitenbach [5] wurden verschiedene Ansätze des Walzprofilierens von Rohren hinsichtlich der Biegefolge diskutiert. Dabei wurde der Radius beispielsweise gleichmäßig im Prozess und über die gesamte Blechbreite verändert, um kontinuierlich zum gewünschten finalen Rohrradius zu gelangen. Alternativ wurde der Prozess so durchgeführt, dass der angestrebte Rohrradius frühzeitig an den Außenkanten des Blechs erreicht wurde und in den weiteren Prozessschritten nach und nach, zur Blechmitte hin, eingestellt wurde. In der Arbeit von Ona [6] wurden vielversprechende Ergebnisse der Rohrherstellung aus hochfestem (2100 MPa) Stahl mit 0,1 mm Dicke und $40 \mathrm{~mm}$ Breite mittels Walzprofilierens erzielt. Unter Verwendung von 21 Walzgerüsten wurde ein vollständig geschlossenes Rohr mit einem Durchmesser von ungefähr $12,7 \mathrm{~mm}$ hergestellt. Die beiden genannten experimentellen Arbeiten zeigen somit, dass die Herstellung vollständig geschlossener Rohre aus hochfesten Werkstoffen im Walzprofilieren möglich ist, dabei aber durchaus eine signifikante Anzahl an Walzgerüsten benötigt wird. In beiden Arbeiten stand die Erzeugung bistabiler Blechstrukturen nicht im Fokus. 
In der Arbeit von Xia et al. [7] wurden numerische Simulationen des mehrstufigen Biegens von Blechen mit einer Dicke von $2 \mathrm{~mm}$ und einer Streckgrenze von ca. $170 \mathrm{MPa}$ in ein zylindrisches Rohr mit der kommerziellen Software LS-DYNA durchgeführt. Die numerische Simulation stimmte gut mit den experimentellen Werten überein. Brunet el al. [8] nutzten neben einem aufwändigeren 3DModell auch ein 2D-FE-Modell um den sich einstellenden Querschnitt beim Walzprofilieren abschätzen zu können. Die beiden numerischen Arbeiten zeigen, dass bereits eine grobe Abschätzung der sich einstellenden Geometrien nach dem Walzprofilieren simulativ möglich ist, wenngleich eine vollständige Abbildung des Gesamtprozesses rechenaufwändig ist.

Ziel dieser Arbeit ist die Abschätzung der Einsetzbarkeit des Walzprofilierens zur Herstellung bistabiler dünner Blechstrukturen, sodass nahezu beliebige Strukturlängen erzeugt werden können. Die zitierten Arbeiten lassen vermuten, dass sowohl simulativ (komplexe Modell, lange Rechenzeiten) als auch experimentell (viele Walzen und Walzgerüste, Führung der Bleche) ein größerer Aufwand zu betreiben ist, um die Nachweisbarkeit der Eignung des Walzprofilierens für die Fertigung vollständig geschlossener bistabiler Rohre führen zu können. Da allerdings die Anzahl notwendiger Walzendurchläufe, die genauen Walzenkonturen und der Einfluss der lokalen Umformzone im Walzprofilieren auf die sich einstellenden Eigenspannungen und letztlich auf die Erreichbarkeit bistabiler Eigenschaften unbekannt sind, liefert die vorliegende Arbeit erste Einschätzungen zu den zuvor genannten Aspekten. Es wird gezeigt, dass durch stark vereinfachte 2D-FE-Modelle eine grobe Abschätzung der Anzahl nötiger Walzendurchläufe sowie die Auslegung der Walzengeometrien möglich ist. Die experimentellen Vorstudien mit nur einem Walzgerüst, weisen zudem nach, dass durch eine Kombination von inkrementellem Gesenkbiegen (1. Fertigungsschritt) und anschließender mehrfacher Verformung des Blechs in nur einem einzelnen Walzprofiliergerüst (2. Fertigungsschritt) bistabile Strukturen herstellbar sind. Die präsentierten Vorarbeiten legen somit nahe, dass das Walzprofilieren geeignet für die Einbringung von Eigenspannungsverläufen zur Erreichung bistabiler Blechstrukturen ist, wenngleich weiterführende Untersuchungen, z.B. zur gegenseitigen Beeinflussung mehrerer Gerüste in einer Walzprofilierstraße, noch ausstehen.

\section{Experimenteller Aufbau und Simulationsmodell}

\subsection{Materialien}

Zur Untersuchung der Erzeugung von bistabilen Eigenschaften durch Walzprofilieren wurden zwei Arten von Materialien mit einer Dicke von 0,2 mm verwendet: Der Federstahl 1.1274 (AISI 1095) und der austenitische Stahl 1.4310 (AISI 301). Diese beiden Materialien wurden aufgrund ihrer hohen Streckgrenze ausgewählt, welche zur Erreichung bistabiler Eigenschaften erforderlich ist [1]. Die mechanischen Eigenschaften der Materialien sind in Tab. 1 gezeigt. Fließkurven aus Zugversuchen und passend gefittete Fließkurven für ein isotropes Verfestigungsgesetz sind in Abb. 1 dargestellt. Die Blechzuschnitte wurden zu $300 \times 300 \mathrm{~mm}^{2}$ für den Federstahl und zu $230 \times 300 \mathrm{~mm}^{2}$ für den weicheren austenitischen Stahl gewählt.

\subsection{Versuchsaufbau}

Die Walzprofilierversuche wurden auf einer Dreistern P3 und zur Reduktion des Versuchsaufwands mit nur einem Walzgerüst durchgeführt. Abb. 2a zeigt die genutzten Walzenpaare mit einem Durchmesser (D) von $280 \mathrm{~mm}$ und Formradien $\left(\mathrm{R}_{\mathrm{f}}\right)$ von 6 bzw. $8 \mathrm{~mm}$. Der kleinere Radius wurde für die Bearbeitung des festeren Federstahls eingesetzt während der größere Radius beim Walzen des austenitischen Stahls zum Einsatz kam. Die Bleche wurden zunächst zwischen den beiden Walzen ausgerichtet und nach jedem Walzschritt manuell in Querrichtung verschoben und neupositioniert (siehe Abb. 2b). Zur besseren Ausrichtung wurden entsprechend der vorgesehenen Anzahl an Walzdurchläufen äquidistante Linien vorab auf dem Blech aufgezeichnet. Die Tab. 2 und 3 zeigen die gewählten Schrittweiten bzw. die nötige Anzahl an Walzdurchläufen für die beiden verwendeten Werkstoffe bzw. Blechformate. Dabei ist zu beachten, dass der erste und letzte Walzdurchlauf einen Abstand von mindestens $25 \mathrm{~mm}$ zum Blechrand aufweist. Aufgrund der geringeren Blechbreite des austenitischen Stahls und der Ausrichtung der längeren Blechseite in Walzrichtung, ergeben sich geringere maximale Walzdurchläufe für diesen Werkstoff. Zusätzlich wurde in unterschiedlichen Versuchsreihen der initiale Zustand des Blechs variiert. Zunächst wurden unverformte Bleche genutzt, welche

Tab. 1 Mechanische Eigenschaften der Materialien und gefittete Parameter $(Q, b)$ für ein isotropes Materialmodell (Abgleich dargestellt in Abb. 1)

\begin{tabular}{llllll}
\hline Stahlsorte & $\begin{array}{l}\text { Elastizitätsmodul, E } \\
{[\mathrm{MPa}]}\end{array}$ & $\begin{array}{l}\text { Initiale Streckgrenze, } \sigma_{0} \\
{[\mathrm{MPa}]}\end{array}$ & $\begin{array}{l}\text { Zugfestigkeit, } \mathrm{R}_{\mathrm{m}} \\
{[\mathrm{MPa}]}\end{array}$ & $\begin{array}{l}\text { Gleichmaßdehnung, } \\
\operatorname{Ag}[\%]\end{array}$ & $\begin{array}{l}\mathrm{Q} \\
{[\mathrm{MPa}]}\end{array}$ \\
\hline 1.4310 & 196.414 & 918 & 1243 & 19,5 & 158,01 \\
1.1274 & 191.230 & 1820 & 1943 & 1,49 & 171,72 \\
\hline
\end{tabular}




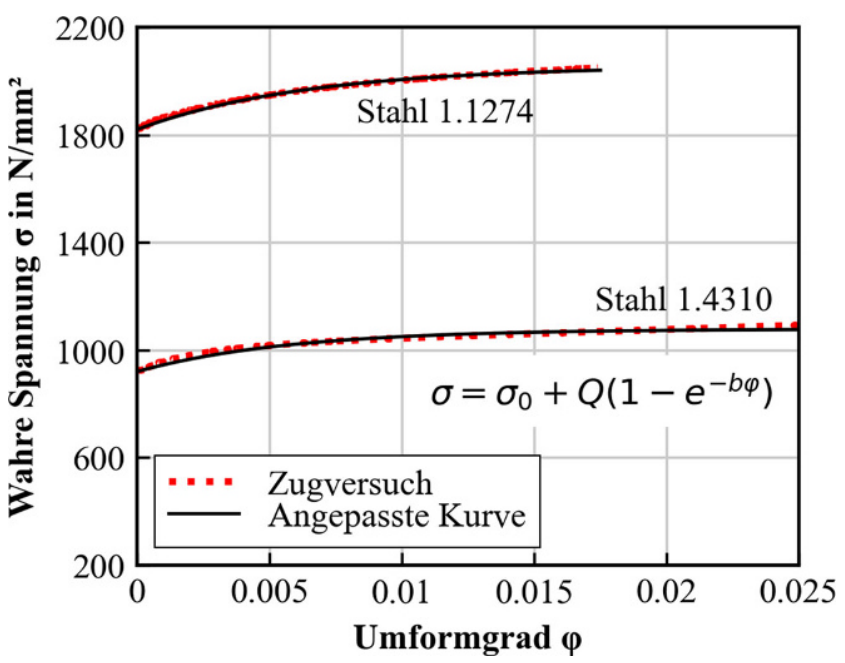

Abb. 1 Fließkurven aus Zugversuchen und gefittete Fließkurven für das verwendete isotrope Verfestigungsgesetz: $\sigma_{0}-$ Streckgrenze, $Q-$ maximale Veränderung der isotropen Fließfläche, $b$ - Änderungsrate der isotropen Fließfläche

lediglich durch den Walzprofilierprozess verformt werden. Hierdurch können keine bistabilen Eigenschaften erreicht werden, aber die Versuche können generell aufzeigen, mit welcher Prozessführung geschlossene Rohre erzeugt werden können. In der zweiten Versuchsreihe wurden die Bleche vorab über einen Biegeprozess, wie in [2] beschrieben, vorverformt. Die Bleche wurden also zunächst inkremen- tell um den Biegeradius $\left(\mathrm{R}_{\mathrm{B}}\right)$ von $6 \mathrm{~mm}$ (für 1.1274) bzw. $8 \mathrm{~mm}$ (für 1.4310) mit einer konstanten Schrittweite von $10 \mathrm{~mm}$ um die kürzere Blechachse gebogen. Der inkrementelle Biegeprozess ist schematisch in Abb. 3 abgebildet. Das anschließende Walzprofilieren (mit der längeren Blechachse in Walzrichtung) wurde mit den gleichen Parametern wie bei den zuvor nicht vorverformten Blechen durchgeführt.

\subsection{Simulation des Walzprofilierprozesses}

Das Walzprofilieren wurde zusätzlich mit einem stark vereinfachten 2D-Modell in Abaqus simuliert, um den Einfluss der Anzahl an Walzdurchläufen auf die erreichbaren Krümmungsradien vorab simulativ abschätzen zu können. Die Vereinfachung des Walzprofilierprozesses in ein 2DBiegemodell wurde als zweckmäßig angesehen, da für die Versuche nur ein Walzgerüst verwendet wird und die Verformungen nacheinander durchgeführt wurden. Das numerische Ersatzmodell ist in Abb. 4 dargestellt. Es besteht aus dem formgebenden Walzenpaar (Kavität in der unteren Walze) und Hilfswerkzeugen. Diese Hilfswerkzeuge sind starr modelliert und übertragen einfache Randbedingungen zur Lagerung des Blechs, während die Walzenpaare nach jedem Umformschritt horizontal versetzt werden. Das verformbare Blech hat eine Dicke von 0,2 $\mathrm{mm}$ und die zugehörigen Materialeigenschaften wurden entsprechend des verwendeten Werkstoffs (vergleiche Tab. 1) ausgewählt. Die
Abb. 2 Skizze der Walzprofilieranlage (a) und Skizze des Ablaufs der Walzprofilierversuche (b) a

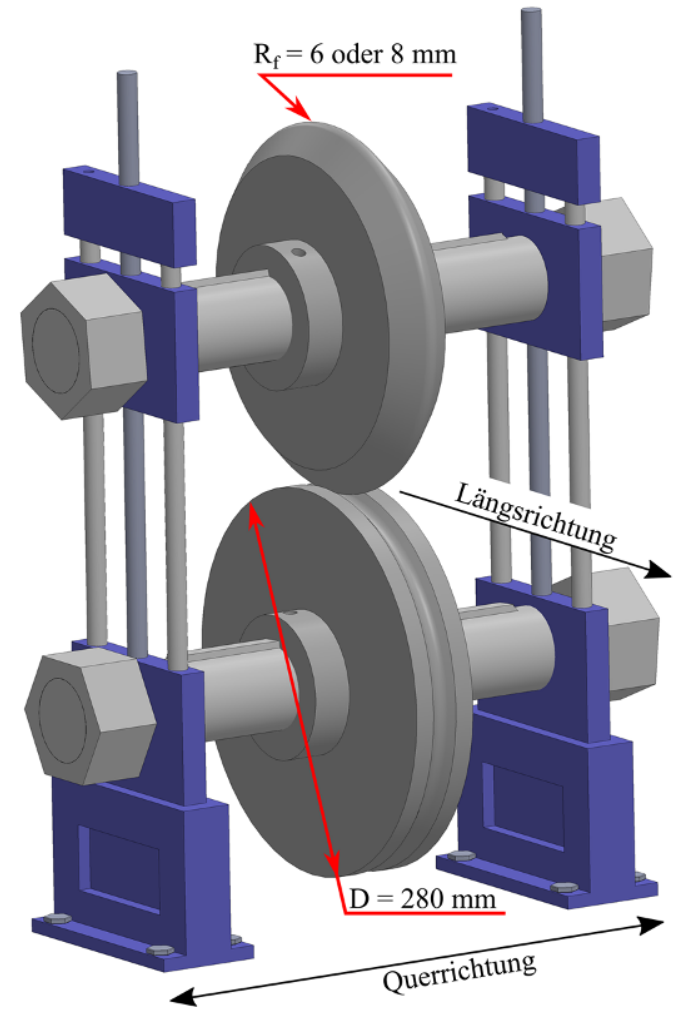

b

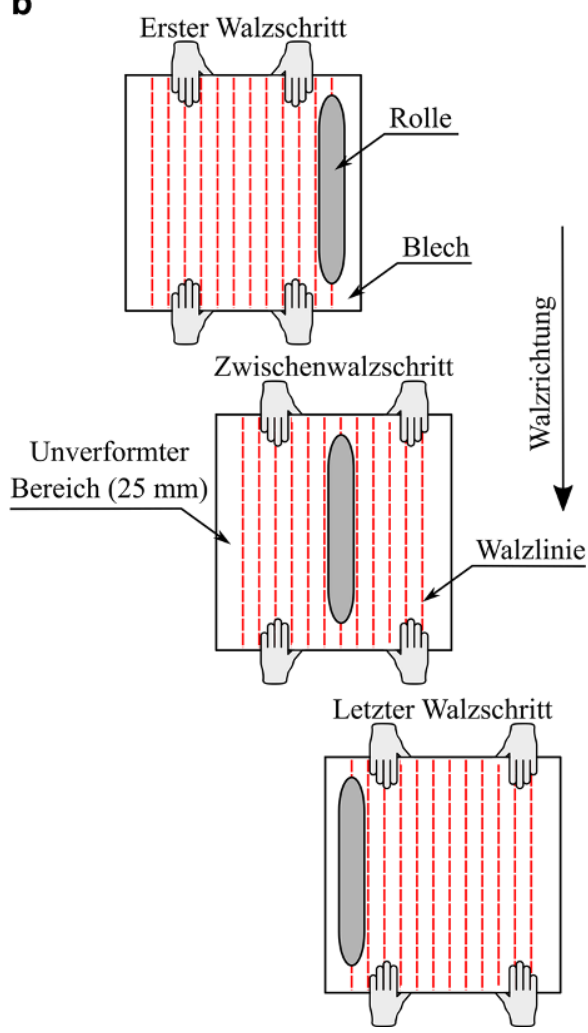


Breite des Blechs beträgt $300 \mathrm{~mm}$ für den Federstahl 1.1274 und $230 \mathrm{~mm}$ für den austenitischen Stahl 1.4310. Aufgrund der Dicke des Blechs weist die Kavität der unteren Walze einen um 0,2 mm größeren Biegeradius als die Oberwalze auf. Der eigentliche Walzschritt wird durch ein vertikales Verschieben der Oberwalze dargestellt, sodass das Blech in der Kavität der unteren Walze gebogen wird. Nach jedem Walzschritt wird das Walzenpaar um die vorgegebene Schrittweite (vergleiche Tab. 2 und 3) horizontal verschoben.

Die Simulationen verwenden einen Penalty-Kontakt mit einem Reibungskoeffizienten von 0,15 zwischen Blech und Walzen. Die Kontakte mit den Hilfswerkzeugen sind reibfrei. Das Blech ist mit 21 CPS4R Elementen (4 Knoten, ebene Spannung, bilinear, reduzierte Integration unter Verwendung von Hourglass-Kontrolle) in Dickenrichtung vernetzt. Die Elementbreite ist so gewählt, dass der Biegeradius des Formwerkzeugs mindestens mit 19 Elementen (bei $\mathrm{R}_{\mathrm{f}}=6 \mathrm{~mm}$ ) bzw. 25 Elementen (bei $\mathrm{R}_{\mathrm{f}}=8 \mathrm{~mm}$ ) des Werkstücks in Kontakt steht.

Tab. 2 Versuchsparameter für den Federstahl $1.1274\left(300 \times 300 \mathrm{~mm}^{2}\right)$ bearbeitet mit Formradius $\mathrm{R}_{\mathrm{f}}=6 \mathrm{~mm}$; Hinweis: Der erste und letzte Walzschritt haben einen Abstand von mindestens $25 \mathrm{~mm}$ zum Blechrand

\begin{tabular}{ll}
\hline Anzahl der Walzschritte & Schrittweite in $\mathrm{mm}$ \\
\hline 12 & 23 \\
13 & 21 \\
14 & 19 \\
15 & 18 \\
16 & 17 \\
17 & 16 \\
18 & 15 \\
19 & 14 \\
20 & 13 \\
21 & 12 \\
23 & 11 \\
26 & 10 \\
\hline
\end{tabular}

Tab. 3 Versuchsparameter für den austenitischen Stahl 1.4310 $\left(230 \times 300 \mathrm{~mm}^{2}\right)$ bearbeitet mit Formradius $\mathrm{R}_{\mathrm{f}}=8 \mathrm{~mm}$; Hinweis: Der erste und letzte Walzschritt haben einen Abstand von mindestens $25 \mathrm{~mm}$ zum Blechrand

\begin{tabular}{ll}
\hline Anzahl der Walzschritte & Schrittweite in $\mathrm{mm}$ \\
\hline 12 & 16 \\
13 & 15 \\
14 & 14 \\
15 & 13 \\
16 & 12 \\
17 & 11 \\
18 & 10 \\
20 & 9 \\
22 & 8 \\
\hline
\end{tabular}

Zur abschließenden Simulation der Rückfederung des Blechs, wurde zusätzlich ein implizites Modell in Abaqus/ Standard verwendet. Dieses Modell enthält ein Blech mit gleicher geometrischer Größe und Netzgröße und berechnet in einem „Static General“-Step die Rückfederung auf Basis der aus der expliziten Simulationen importierten Spannungs- und Dehnungswerte nach der Abbildung aller Umformschritte im Walzprofilieren.

\section{Ergebnisse und Diskussion}

Durch das Walzprofilieren mit einer unterschiedlichen Anzahl an Walzschritten ändert sich die sich einstellende Geometrie der Struktur nach der Rückfederung auf Basis der eingebrachten Eigenspannungen. Nach jedem Walzprofilierversuch wurde die Krümmung des verformten Stahlblechs auf ein Blatt Papier übertragen. Anschließend wird der Krümmungsradius der Struktur aus der Sehnenlänge und dem Abstand der Sehne zur Oberfläche der Kreisgeometrie entsprechend Abb. 5 berechnet.

\subsection{Simulation des Walzprofilierprozesses}

Abb. 6 zeigt beispielhaft die simulierten Strukturen nach dem Walzprofilieren für den Federstahl 1.1274 nach 12 und 26 Walzschritten. Es ist klar zu erkennen, dass ein vollständig geschlossenes Rohrprofil $(\mathrm{r}<40 \mathrm{~mm})$ erst nach deutlich mehr als 12 Walzschritten erreicht werden kann. Die Anzahl benötigter Profiliergerüste kann somit schon einmal grob abgeschätzt werden. Abb. 7 zeigt die simulierten Krümmungsradien der erzeugten Strukturen in Abhängigkeit der Anzahl an Walzdurchläufen. Zusätzlich ist für die unterschiedlichen Blechformate derjenige Krümmungsradius markiert, unterhalb dessen ein vollständig geschlossenes Rohr vorliegt. Beim Federstahl 1.1274 nimmt der Rückfederungsradius mit steigender Anzahl an Walzschritten linear ab. Der austenitische Stahl 1.4310 weist ab etwa 16 Walzschritten keine weitere Veränderung des Krümmungsradius, trotz weiteren Walzschritten, auf. Dies kann dadurch begründet werden, dass bei der Verwendung eines Biegeradius von $8 \mathrm{~mm}$ und einer Blechbreite von $230 \mathrm{~mm}$ (Stahl 1.4310) nach 16 Walzschritten keine unverformten Bereiche mehr zwischen den einzelnen Walzspuren vorliegen und eine homogene Verformung über die Blechbreite erreicht ist. Im Gegensatz dazu sind beim Biegeradius von $6 \mathrm{~mm}$ und einer Blechbreite von $300 \mathrm{~mm}$ (Stahl 1.1274) auch nach 23 Walzschritten noch unverformte Blechbereiche zwischen den einzelnen Walzspuren vorherrschend.

Abb. 8 zeigt die Eigenspannungsverteilung über die Blechdicke für die Stähle 1.1274 und 1.4310 nach erfolgter Rückfederung. Die Eigenspannungen wurden in einem Blechbereich ausgewertet, in dem das Blech in di- 


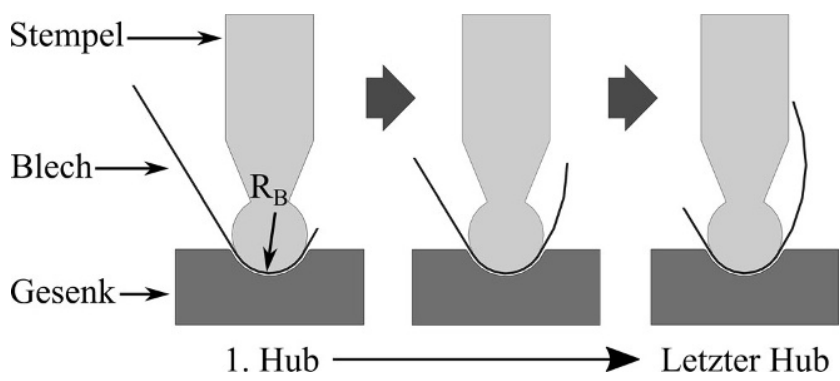

Abb. 3 Skizze des inkrementellen Biegeprozesses

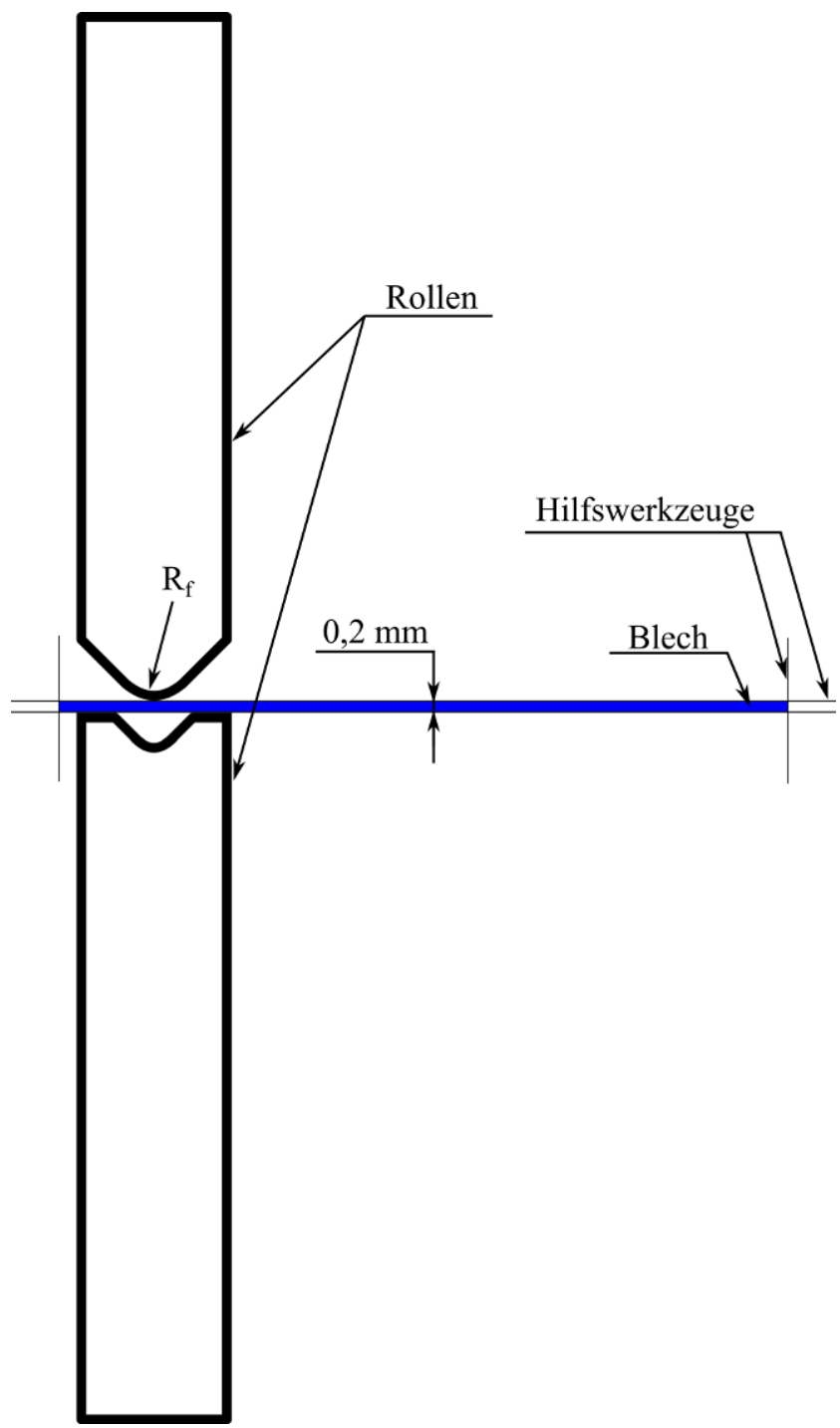

Abb. 4 Numerisches 2D-Ersatzmodell des Walzformprozesses

rektem Kontakt mit den Walzen war. Der Grund für den Unterschied im Krümmungsradius über die Anzahl der Walzschritte ist die unterschiedliche Verteilung der Eigenspannungen in Querrichtung. Die resultierenden Spannungen in $\mathrm{x}$-Richtung sind vergleichbar mit denjenigen die in [2] über das inkrementelle Gesenkbiegen erreicht wurden.

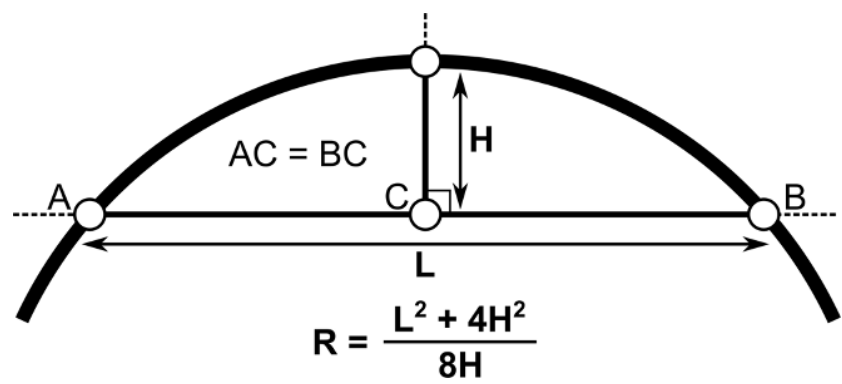

Abb. 5 Bestimmung des Krümmungsradius $\mathrm{R}$

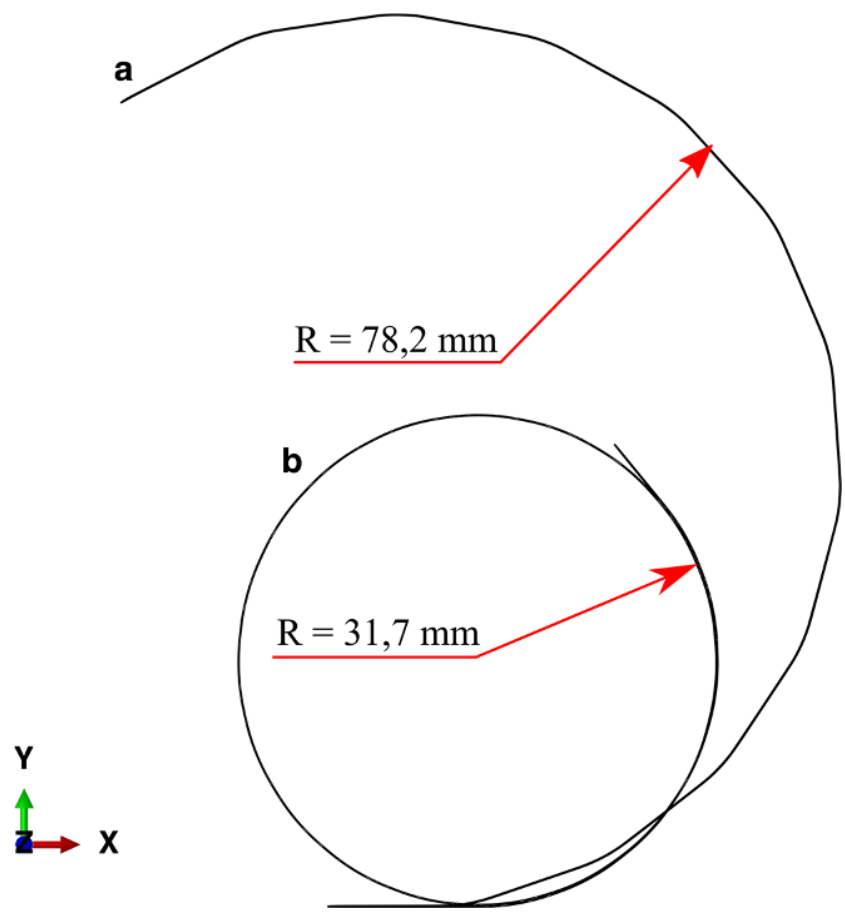

Abb. 6 Simulierte Strukturen nach dem Walzprofilieren für den Federstahl 1.1274 nach 12 (a) und 26 (b) Walzschritten

Das Walzprofilieren kann also genutzt werden, um ähnliche Eigenspannungsverteilungen zu erreichen.

\subsection{Walzprofilierversuche an unverformten Blechen}

Die simulierten Ergebnisse können aufgrund der starken Vereinfachungen des FE-Modells nur einen groben Einblick in die Abhängigkeiten beim Walzprofilieren geben. Daher zeigt Abb. 7 zusätzlich die zugehörigen experimentellen Ergebnisse an zuvor unverformten Blechen. Trotz der bereits angeführten Vereinfachungen des Simulationsmodells ist in den experimentellen Ergebnissen die gleiche Abhängigkeit wie in den Simulationen zu erkennen: Mit abnehmender Schrittweite nimmt auch der Radius der endgültigen Krümmung des Blechs ab. Es ist jedoch zu beachten, dass die Ergebnisse der Experimente generell oberhalb der simulierten Krümmungsradien liegen. Für die Abweichun- 

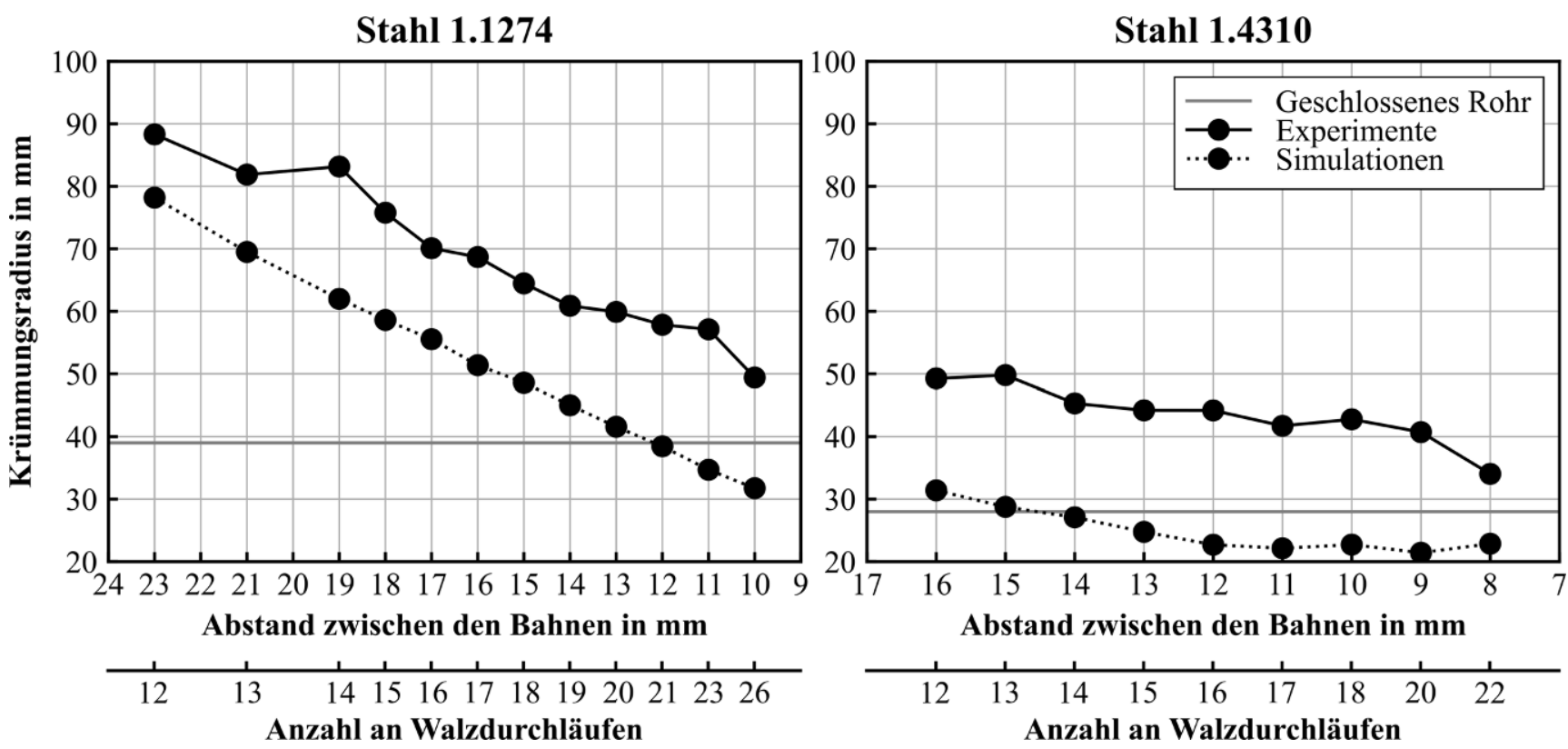

Abb. 7 Simulierte und experimentelle Krümmungsradien der erzeugten Strukturen in Abhängigkeit der Anzahl an Walzdurchläufen

gen zwischen Experimenten und Simulationen ist neben den Vereinfachungen des FE-Modells auch das herausfordernde Handling der Bleche in den Experimenten (genaue Positionierung der Walzen auf dem Blech, paralleles Durchlaufen des Walzschritts auf der zuvor markierten Bahn, etc.) verantwortlich. Hierdurch lassen sich vermutlich auch einzelne Ausreißer innerhalb der Versuchsreihen, z.B. der erhöhte Krümmungsradius für 1.1274 bei 14 Walzdurchläufen, erklären. In Abb. 9 sind beispielhaft die walzprofilierten Bleche mit minimal erreichtem Krümmungsradius für das jeweilige Material dargestellt.

\subsection{Walzprofilierversuche mit vorgebogenen Blechen}

Durch eine vorherige Biegung der Bleche in einem inkrementellen Biegeprozess mit Biegeradien von $6 \mathrm{~mm}$ für den Federstahl 1.1274 und $8 \mathrm{~mm}$ für den austenitischen Stahl 1.4310 (mit einem Abstand zwischen den Biegungen von $10 \mathrm{~mm}$ ) und eine anschließende Bearbeitung im Walzprofilieren können bistabile Strukturen erzeugt werden. In Abb. 10 sind ausgewählte bistabile Bleche beider Materialien gezeigt, die durch die Abfolge von inkrementellem Biegen und Walzprofilieren hergestellt wurden. Die experimentell erreichten Krümmungsradien der bistabilen Strukturen sind für beide stabilen Geometrien und in Abhängigkeit der gewählten Anzahl an Walzdurchläufen in Abb. 11 für beide verwendeten Stähle dargestellt. In den Walzfällen, in denen keinen bistabilen Eigenschaften erreicht wurden, ist nur der Krümmungsradius des monostabilen Zustands eingezeichnet.

Aus Abb. 11 wird ersichtlich, dass der Krümmungsradius des ersten stabilen Zustands mit abnehmender Schrittweite nahezu linear abnimmt. Für den zweiten stabilen $\mathrm{Zu}$ - a

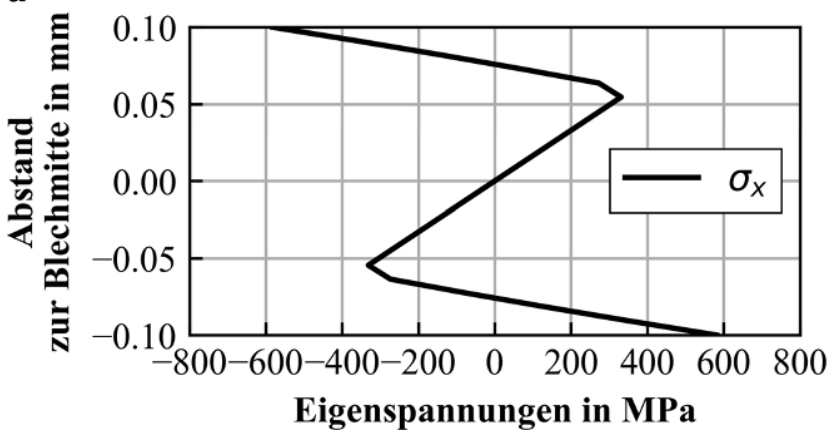

b

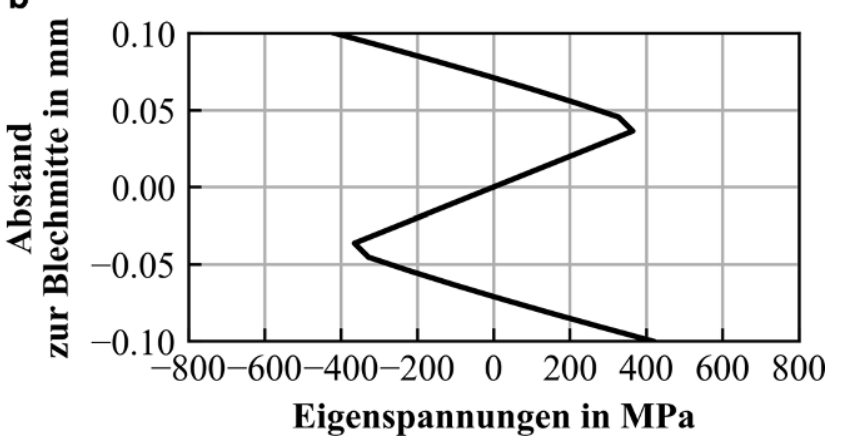

Abb. 8 Simulierte Eigenspannungen in x-Richtung über die Blechdicke für die Stähle 1.1274 (a) und 1.4310 (b) 

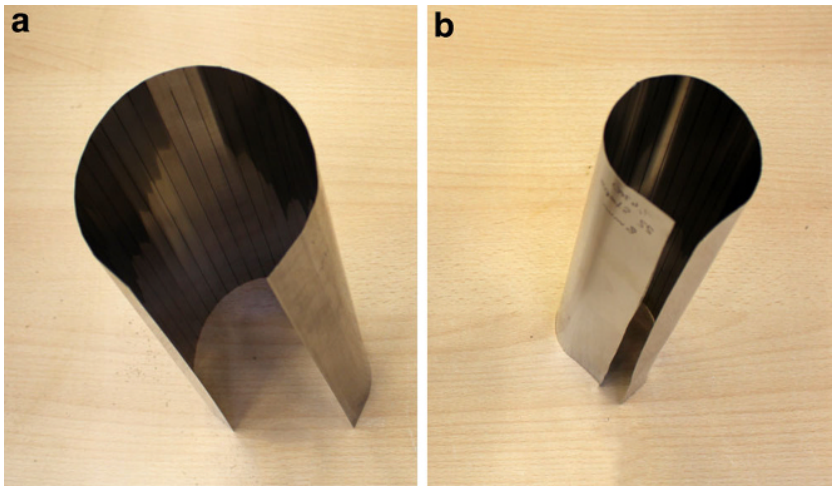

Abb. 9 Walzprofilierte Bleche aus 1.1274 nach 26 Walzschritten (a) und 1.4310 nach 22 Walzschritten (b)

stands ergibt sich ebenfalls eine signifikante Abhängigkeit von der Schrittweite beim Walzprofilieren. Auch in dieser experimentellen Versuchsreihe lassen sich Diskontinuitäten und Unregelmäßigkeiten der Werte, z. B. zwischen 15 und 18 Walzdurchläufen, vermutlich durch die Herausforderungen bei der Blechpositionierung und der genauen Durchführung des Walzschritts entlang der vorgezeichneten Bahn erklären.

\section{Zusammenfassung und Ausblick}

Die Ergebnisse der Simulation zeigen bereits die Möglichkeit auf, Rohrgeometrien für dünne hochfeste Bleche im
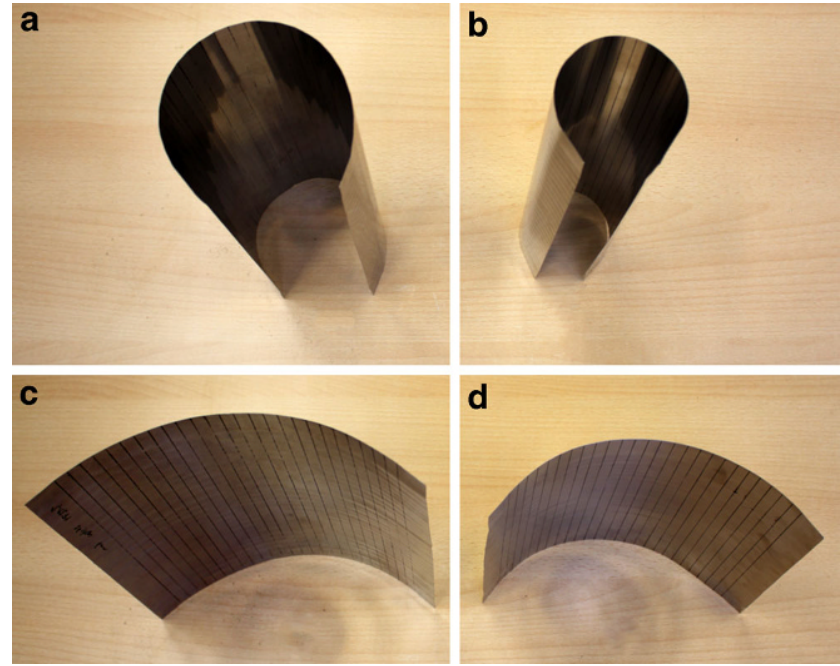

Abb. 10 Bistabile Bleche, hergestellt durch inkrementelles Biegen und anschließendes Walzprofilieren. Der Federstahl 1.1274 nach 26 Walzschritten im ersten (a) und zweiten (c) stabilen Zustand und der austenitische Stahl 1.4310 nach 22 Walzschritten im ersten (b) und zweiten (d) stabilen Zustand

Walzprofilieren zu erzeugen. Eine Bistabilität der Strukturen tritt dabei noch nicht auf. Die experimentellen Ergebnisse zeigen gleiche Tendenzen, weisen aber einen gewissen Versatz gegenüber den Simulationen auf. Zur Erzeugung einer fast geschlossenen Rohrstruktur ist eine große Anzahl von Walzschritten erforderlich: 26 Walzschritte für den Federstahl 1.1274 bei einer Blechbreite von $300 \mathrm{~mm}$

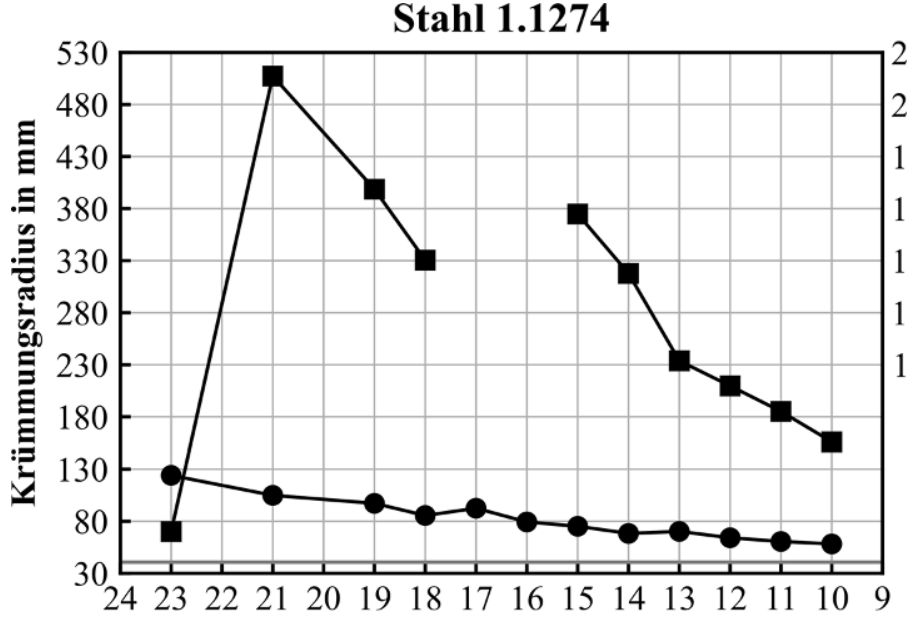

Abstand zwischen den Bahnen in mm

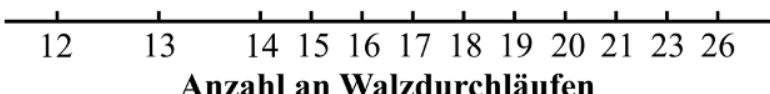

Stahl 1.4310

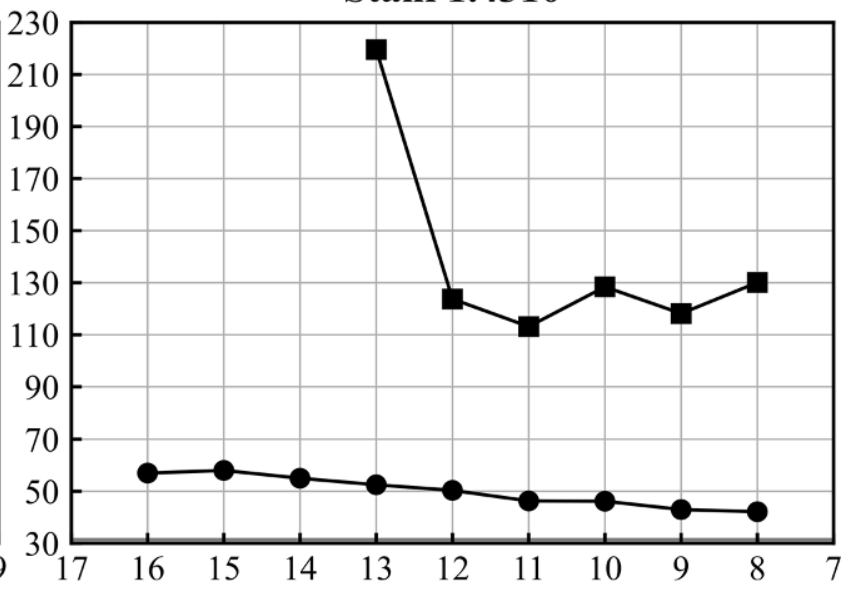

Abstand zwischen den Bahnen in mm

\begin{tabular}{lllllllll}
\hline 12 & 13 & 14 & 15 & 16 & 17 & 18 & 20 & 22
\end{tabular}

$\longrightarrow$ Geschlossenes Rohr $\longrightarrow$ Erster stabiler Zustand $\rightarrow-$ Zweiter stabiler Zustand

Abb. 11 Experimentell erreichte Krümmungsradien der erzeugten Strukturen in den beiden stabilen Lagen in Abhängigkeit der Anzahl an Walzdurchläufen bei der Verwendung vorverformter Bleche 
und 22 Walzschritte für den austenitischen Stahl 1.4310 bei einer Blechbreite von $230 \mathrm{~mm}$.

Durch eine vorgeschaltete Verformung der Bleche mittels inkrementellen Biegens und ein anschließendes Walzprofilieren können bistabile Eigenschaften für beide Blechmaterialien eingestellt werden. Es muss jedoch auch hier eine hohe Anzahl von Walzschritten durchgeführt werden, um eine nahezu vollständig geschlossene Rohrgeometrie zu erreichen.

Zur weiteren Absicherung der Ergebnisse und zur genaueren Vorhersage der Bistabilität und der erreichten Krümmungsradien muss das Handling der Bleche in den experimentellen Versuchen weiter optimiert werden. Des Weiteren steht noch die experimentelle Abbildung eines realen Walzprofilierprozesses mit mehreren, gleichzeitig im Eingriff befindlichen, Walzgerüsten aus. Darüber hinaus sollten rechenaufwändigere dreidimensionale FE-Modelle genauere Vorberechnungen der Prozessrouten ermöglichen. Neben dem größeren Rechenaufwand sind allerdings auch hier Herausforderungen hinsichtlich des Blechhandlings bzw. der Lagerung des Blechs während der Umformung zu erwarten. Nichtsdestotrotz deuten die vereinfachten experimentellen und simulativen Arbeiten bereits jetzt auf eine Einsetzbarkeit des Walzprofilierens hin.

Förderung Das Projekt wurde durch die Deutsche Forschungsgemeinschaft (DFG) - Projekt HI 790/57-2, No 374688658 im Rahmen des SPP 2013 gefördert. Die Autoren bedanken sich für die Förderung.

Funding Open Access funding enabled and organized by Projekt DEAL.

Open Access Dieser Artikel wird unter der Creative Commons Namensnennung 4.0 International Lizenz veröffentlicht, welche die Nutzung, Vervielfältigung, Bearbeitung, Verbreitung und Wiedergabe in jeglichem Medium und Format erlaubt, sofern Sie den/die ursprünglichen Autor(en) und die Quelle ordnungsgemäß nennen, einen Link zur Creative Commons Lizenz beifügen und angeben, ob Änderungen vorgenommen wurden.

Die in diesem Artikel enthaltenen Bilder und sonstiges Drittmaterial unterliegen ebenfalls der genannten Creative Commons Lizenz, sofern sich aus der Abbildungslegende nichts anderes ergibt. Sofern das betreffende Material nicht unter der genannten Creative Commons Lizenz steht und die betreffende Handlung nicht nach gesetzlichen Vorschriften erlaubt ist, ist für die oben aufgeführten Weiterverwendungen des Materials die Einwilligung des jeweiligen Rechteinhabers einzuholen.

Weitere Details zur Lizenz entnehmen Sie bitte der Lizenzinformation auf http://creativecommons.org/licenses/by/4.0/deed.de.

\section{Literatur}

1. Kebadze E, Guest S, Pellegrino S (2004) Bistable prestressed shell structures. Int J Solids Struct 41:2801-2820

2. Pavliuchenko P, Teller M, Grüber M et al (2019) Production of bistable fully closed metallic shells by introducing residual stresses during bending processes. Prod Eng Res Dev 13:201-209

3. Pavliuchenko P, Teller M, Grüber M, Hirt G (2019) A semianalytical model for the determination of bistability and curvature of metallic cylindrical shells. J Manuf Mater Process 3(1):22

4. Bralla JG (2000) Design for manufacturability handbook. McGrawHill, New York

5. Groche P, Breitenbach G (2008) Roll forming strategies for welded tubes. Steel Res Int 79:40-46

6. Ona H (2004) Cold roll forming for high tensile strength steel sheet proposition on forming of thin spring steel sheet pipe. J Mater Process Technol 153/154:247-252

7. Xia M, Yan Q, Zuo D, Xie J (2011) An investigation on multistage bending of blank sheet into cylindrical tube by experiment and numerical simulation. Int J Adv Manuf Technol 53:145-155

8. Brunet M, Mguil S, Pol P (1998) Modelling of a roll-forming process with a combined 2D and 3D FEM code. J Mater Process Technol 80/81:213-219 